\title{
A162 PROTEIN ARRAY SCREENING REVEALS IGA AUTOANTIGENICITY PATTERNS PREDICTING ANTI-TNF $\alpha$ THERAPY RESPONSE IN RHEUMATOID ARTHRITIS PATIENTS
}

Zoltán Konthur, ${ }^{1}$ Katja Köpke, ${ }^{2}$ Annette Poch-Hasnek, ${ }^{1}$ Katja Köpke, ${ }^{2}$ Hans Lehrach, ${ }^{1}$ Gerd-Rüdiger Burmester, ${ }^{2}$ Karl Skriner ${ }^{2}$ Department of Vertebrate Genomics, Max Planck Institute for Molecular Genetics, Berlin, Germany; ${ }^{2}$ Department of Rheumatology and Clinical Immunology, Humboldt University and Free University, Berlin, Germany

\subsection{6/ard.2010.149013.5}

Background One third of rheumatoid arthritis (RA) patients treated with biologicals targeting tumour necrosis factor $\alpha$ (TNF $\alpha$ ) are therapy non-responders. The authors investigated the differences in seroreactivity of patients responding and not responding to TNF therapies prior and after therapy to deduce diagnostically applicable autoantigenicity patterns.

Methods Screening with patient sera were conducted on protein macroarrays consisting of 37.830 unique putative expression clones. Response patterns of different Ig classes were recorded and bioinformatically evaluated enabling them to deduce a set of proteins, which allow to distinguish between therapy responders and non-responders. Next, selected candidates were expressed recombinantly in Escherichia coli, purified and further stratified with larger patient cohort in ELISA responder group.

Results Comparative analysis of macroarray results with sera from responders and non-responders to anti-TNF drug revealed a more than 30 -fold higher number of autoantigens targeted by high titres of IgA autoantibodies in non-responders compared to responders (221 vs 6). More detailed analyses suggest that with five autoantigens found to be common in all individual non-responders to anti-TNF $\alpha$ treatment, a reduced number of antigens might be sufficient to predict non-responsiveness. Pretreatment sera from patients with diagnosis of RA based on the ACR classification criteria who were initiated on therapy with TNF $\alpha$ inhibitors were analysed with three markers from the biomarker set of highest priority (RAB11B, PPP2R1A, KPNB1) using an ELISAs assay. In total, analyses of 69 patients were carried out, of which 13 were clearly defined as Responder and 8 were clearly defined as non-responder. Of these, already 5 (62.5\%) nonresponders could clearly be identified with already three markers from biomarker set of highest priority (RAB11B, PPP2R1A, KPNB1). None of the Responder or Intermediate Responder gave any signal on said markers on IgA-level. The remaining 48 patient samples are derived prior treatment 
with anti-TNF $\alpha$ inhibitors and were blinded. According to published studies, $20-25 \%$ of RA patients treated with TNF $\alpha$ inhibitors are non-responders: Hence the authors expect $\sim 10$ patients to be non-responder. Within this set, five patients $(50 \%)$ showed clear IgA response to three markers from biomarker set of highest priority (RAB11B, PPP2R1A, KPNB1). Furthermore, with five autoantigens common in all individual non-responders to anti-TNF $\alpha$ treatment, a reduced number of antigens may be sufficient to predict nonresponsiveness.

Conclusion These data suggest that non-response to antiTNF $\alpha$ biologicals might be predicted based on frequency and magnitude of autoantibodies of the IgA class IgA-producing mucosal B cells might be important for disease persistence in anti-TNF $\alpha$ non-responders. 